\title{
Stellar fluxes as probes of convection in stellar atmospheres
}

\author{
Barry Smalley \\ Astrophysics Group, Keele University, Staffordshire ST5 5BG, United Kingdom \\ email: bs@astro.keele.ac.uk
}

\begin{abstract}
Convection and turbulence in stellar atmospheres have a significant effect on the emergent flux from late-type stars. The theoretical advancements in convection modelling over recent years have proved challenging for the observers to obtain measurements with sufficient precision and accuracy to allow discrimination between the various predictions. An overview of the current observational techniques used to evaluate various convection theories is presented, including photometry, spectrophotometry, and spectroscopy. The results from these techniques are discussed, along with their successes and limitations. The prospects for improved observations of stellar fluxes are also given.
\end{abstract}

Keywords. Techniques: photometric, techniques: spectroscopic, stars: atmospheres, convection

\section{Introduction}

The gross properties of a star, such as broad-band colours and flux distributions, are significantly affected by the effects of convection in stars later than mid A-type. Consequently, our modelling of convection in stellar atmosphere models can significantly alter our interpretation of observed phenomena. By comparison with stars of known $T_{\text {eff }}$ and/or $\log g$ (the fundamental stars), we can evaluate different treatments of convection in model atmosphere calculations.

\section{Photometry}

Photometric indices are a fast and efficient method for determining approximate atmospheric parameters of stars. For the commonly-used Strömgren uvby system a vast body of observational data exists which can be used to estimate parameters using calibrated model grids (e.g. Moon \& Dworetsky 1985, Smalley \& Dworetsky 1995). Conversely, knowing atmospheric parameters from other methods, allows observed colours to be compared to model predictions. This method has been used to compare various treatments of stellar convection.

The effects of convection on the theoretical uvby colours of A, F, and G stars was discussed by Smalley \& Kupka (1997), who compared the predicted colours for the Canuto \& Mazzitelli (1991), Canuto \& Mazzitelli (1992) (CM) model with that from the standard Kurucz (1993) mixing-length theory (MLT) models with and without "approximate overshooting". Comparison against fundamental $T_{\text {eff }}$ and $\log g$ stars revealed that the CM models gave better agreement than MLT without overshooting. Models with overshooting were clearly discrepant. This result was further supported by stars with $T_{\text {eff }}$ obtained from the Infrared Flux Method (IRFM) and $\log g$ from stellar evolutionary models. 


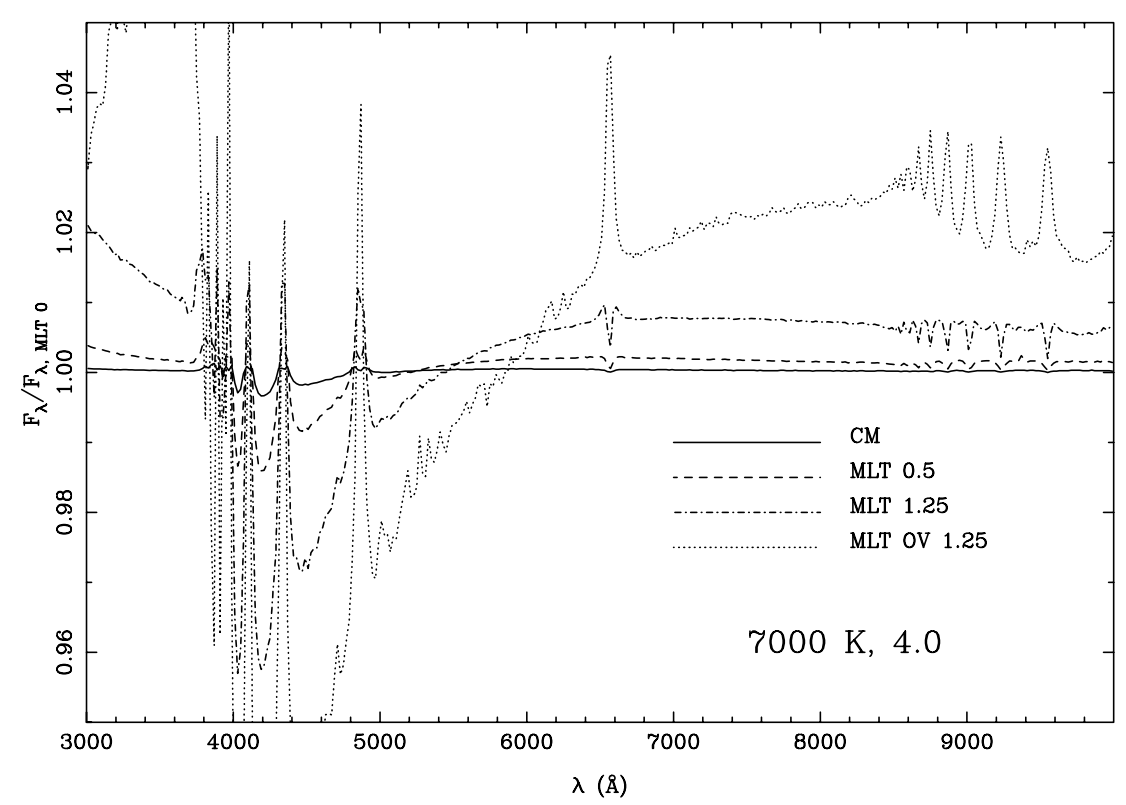

Figure 1. Fluxes for solar-composition $T_{\text {eff }}=7000 \mathrm{~K}, \log g=4$ models with CM and MLT $\left(l / H_{\mathrm{p}}=0.5\right.$ and 1.25$)$, compared to that for a model with zero convection. Note that the region $4000 \sim 5000 \AA$ is especially sensitive and the effect of overshooting is considerable.

\section{Fluxes}

The observed stellar flux distribution is influenced by the effects of convection on the atmospheric structure of the star. As we have seen with photometric colours, these effects have a clearly observable signature (see Fig. 1). In their discussion of convection Lester et al. (1982) presented model stellar atmospheres using a modified mixing-length theory. They found small, systematic differences in the optical fluxes. Their figures also demonstrate that convection can have a measurable effect on stellar fluxes. Hence, high precision stellar flux measurements will provide significant and useful information on convection.

Unfortunately, very little high-precision stellar spectrophotometry exists. This situation will be rectified, once the ASTRA Spectrophotometer (see below) begins operation. This will allow spectrophotometry to be added to our observational diagnostic toolkit.

\section{Balmer Profiles}

The temperature sensitivity of Balmer lines makes them an excellent diagnostic tool for late A-type stars and cooler. The $H \alpha$ and $H \beta$ profiles behave differently due to convection: $H \alpha$ is significantly less sensitive to mixing-length than $H \beta$ (van't Veer \& Mégessier 1996). Both profiles are affected by the presence of overshooting. Since $H \alpha$ is formed higher in the atmosphere than $H \beta$, Balmer lines profiles are a very good depth probe of stellar atmospheres. Balmer profiles are also affected by microturbulence, metallicity and, for hotter stars, surface gravity (Heiter et al. 2002).

In their comparison of Balmer line profiles, Gardiner et al. (1999) found that both CM and MLT without overshooting gave satisfactory agreement with fundamental stars. Overshooting was again found to be discrepant. In addition, Gardiner et al. (1999) found 
evidence for significant disagreement between all treatments of convection for stars with $T_{\text {eff }}$ around $8000 \sim 9000 \mathrm{~K}$. Subsequently, Smalley et al. (2002) reviewed this region using binary systems with known $\log g$ values and their revised fundamental $T_{\text {eff }}$ values of the component stars. They found that the discrepancy found was no longer as evident. However, this region was relatively devoid of stars with fundamental values of both $T_{\text {eff }}$ and $\log g$. Further fundamental stars are clearly required in this region.

\section{The ASTRA Spectrophotometer}

The Automated Spectrophotometric Telescope Research Associates (ASTRA) have developed a cassegrain spectrophotometer and its automated $0.5-\mathrm{m} \mathrm{f} / 16$ telescope. They are being integrated at the Fairborn Observatory near Nogales, Arizona. Scientific observations are expected to begin in 2007 (Adelman et al. 2007, Smalley et al. 2007).

In an hour the system will obtain $\mathrm{S} / \mathrm{N}=200$ (after correction for instrumental errors) observations of stars as faint as 9.5 mag. The spectrograph uses both a grating and a cross-dispersing prism to produce spectra from both the first and the second orders simultaneously. The square 30 arc second sky fields for each order do not overlap. The resolution is $7 \AA$ in second and $14 \AA$ in first order. The wavelength range is of approximately $\lambda \lambda 3300-9000$.

\section{Conclusions}

The effects of convection on the stellar atmospheric structure can be successfully probed using a variety of observational diagnostics (Smalley 2004). The combination of photometric colours and Balmer-line profiles has given us a valuable insight into the nature of convection in stars. High quality observations that are currently available and those that will be in the near future, will enable further refinements in our theoretical models of convection and turbulence in stellar atmospheres.

\section{References}

Adelman, S.J., Gulliver, A.F., Smalley, B., Pazder, J.S., Younger, P.F., Boyd, L.J., Epand, D. \& Younger, T. 2007, in: C. Sterken (ed.), The Future of Photometric, Spectrophotometric and Polarimetric Standardization, (San Francisco: ASP), in press.

Canuto, V.M. \& Mazzitelli, I. 1991, ApJ 370, 295

Canuto, V.M. \& Mazzitelli, I. 1992, ApJ 389, 724

Gardiner, R.B., Kupka, F. \& Smalley, B. 1999, A\&A 347, 876

Heiter, U., Kupka, F., van't Veer-Menneret, C., Barban, C., Weiss, W.W., Goupil, M.-J., Schmidt, W., Katz, D. \& Garrido, R. 2002, A\&A 392, 619

Kurucz, R.L. 1993, Kurucz CD-ROM 13: ATLAS9, SAO, Cambridge, USA

Lester, J.B., Lane, M.C. \& Kurucz, R.L. 1982, ApJ 260, 272

Moon, T.T. \& Dworetsky, M.M. 1985, MNRAS 217, 305

Smalley, B. 2004, in J. Zverko, J. Ziznovsky, S.J. Adelman \& W.W. Weiss (eds.), The A Star Puzzle, Proc. IAU Symposium No. 224 (Cambridge University Press), p. 131

Smalley, B. \& Dworetsky, M.M. 1995, A\&A 293, 446

Smalley, B. \& Kupka F. 1997, A\& A 328, 439

Smalley, B., Gardiner, R.B., Kupka, F. \& Bessell, M.S. 2002, A\&A 395, 601

Smalley, B., Gulliver, A.F. \& Adelman, S.J. 2007, in: C. Sterken (ed.), The Future of Photometric, Spectrophotometric and Polarimetric Standardization, (San Francisco: ASP), in press.

van't Veer, C. \& Mégessier, C. 1996, A\&A 309, 879 